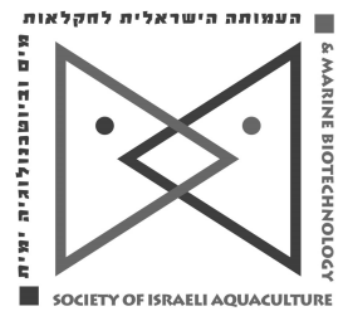

The IJA appears exclusively as a peer-reviewed on-line open-access journal at http://www.siamb.org.il. To read papers free of charge, please register online at registration form.

Sale of IJA papers is strictly forbidden.

\title{
Epidemiological Survey of Grass Carp (Ctenopharyngodon idella) Reovirus in South China, and Genetic Variations of VP6 gene
}

\section{Ying Yang, Zhongqin Peng, Hua Li, Shuwen Tan, Haiyi Yu, Hui Yu*}

School of Life Science and Engineering, Foshan University, Foshan, Guangdong 528231, China

Keywords: Grass carp reovirus; virus isolates; genetic variation; identification; immunogenicity

\begin{abstract}
To study the molecular biology characteristics, phylogenetic relationship, and status quo of grass carp reovirus (GCRV) prevalent isolates, we conducted an epidemiological survey in South China from 2012 to 2016. We sampled 126 fish collected from five provinces. Eight strains of GCRV were isolated and examined in order to understand their genetic characteristics and evolution regularity. The results showed three genotypes of GCRV, of which. Genotype II was found to be the prevalent strain in South China. SDS-PAGE electrophoresis further suggested that all viruses possess 11 segments of dsRNA, which have typical characteristics of GCRV. Based on amino acid sequence phylogenetic tree of VP6 gene, all eight isolates belonged to genotype II and had significant variations compared to genotype I. Analysis of amino acid sequence showed that these two genotypes of GCRV had no immunological cross-reactions. These results indicated that genotype II GCRV is an extensive pandemic, and revealed new genetic diversity in China.
\end{abstract}

\footnotetext{
* Corresponding author: Tel/fax +86-757-86678587. E-mail: 313151361@qq.com
} 
Yang et al.

\section{Introduction}

Genus Aquareovirus, a member of the family Reoviridae, generally causes infection in aquatic animals such as bony fish and shellfish. To date, more than 50 Aquareoviruses have been isolated throughout the world, but only a few of them have been closely investigated, i.e. grass carp reovirus (GCRV) and Striped bass reovirus (SBRV), which belong to different species in this genus (Iwanowicz et al. 2016). GCRV, the most pathogenic Aquareovirus, first isolated in China in 1983, was recognized by the International Committee on Taxonomy of Viruses (ICTV) as a species belonging to the genus Aquareovirus, AQRV-C (Zeng et al. 2017). As a member of the family Reoviridae, GCRV is the causative agent of grass carp hemorrhage, which is the most severe viral disease in China (Liang et al. 2016). The virion has a nonenveloped double capsid shell with an icosahedral morphological structure and consists of 11 segments of double stranded RNA (Tian et al. 2013). While a significant amount of structural and molecular information is available about GCRV (Wang et al. 2013; Huan et al. 2014), knowledge about the variations among different isolates is limited.

Grass carp (Ctenopharyngodon idella) is an important freshwater aquaculture species widely cultured in China and other Asian countries. with an annual production of over 1 million tons. However, fingerling and yearling grass carp are seriously threatened by GCRV, which causes severe hemorrhages with up to $80 \%$ mortality (Jia et al. 2014). Currently, there are no effective treatment methods against GCRV infection, the immunization schedule based on preventive strategies is especially important. To scientifically guide immunization, the epidemiology and variations of this virus need to be elucidated. After grass carp were vaccinated with GCRV, humoral immune response against the VP6 and other structural proteins of this virus played important roles in the protection against grass carp hemorrhage (Bo et al. 2014). The epidemic status and genetic diversity of GCRV in recent years have been explored in this study.

\section{Materials and Methods}

Sample collection and detection. 126 clinical samples were detected by RT-PCR, which were collected from five provinces in South China from 2012 to 2016, a pair of primer 1 were designed based on GCRV GD-108 (108jcF: 5'- GTTCCTGTCGTGGCTGGTAT -3', 108jCR: 5'- GCCATTTGCAGAACTCCATT -3'), and a pair of primer 2 were designed according to GCRV873 (873jcF: 5'- CTCATGCTGCTAACGGTGTCT -3', 873jcR: 5'GCGACTGTGGCACCATCAA -3'). Eight typical samples were selected according to geographic distribution, which included Guangdong (3), Hubei (2), Hunan (1), Jiangxi (1) and Jiangsu (1) virus strains. All tissues were homogenized with DMEM, freeze-thawed three times, and centrifuged at $12,000 \times \mathrm{g}$ for $15 \mathrm{~min}$. The supernatant was sieved through a $0.22 \mathrm{~mm}$ filter and injected into Ctenopharyngodon idellus kidney-CIK cell monolayers. The cells were incubated at $28^{\circ} \mathrm{C}$ for 3-7 days and examined for cytopathic effects (CPE) daily. The cell was continuously passaged to the next generation using the conventional method. The 3rd and 4th generations were used for grass carp artificial infection, viral purification, and RNA isolation. Healthy grass carp (length $8-12 \mathrm{~cm}$ ) were artificially infected using intraperitoneal injection of $100 \mu$ cell suspension diluted by 0.65 $\% \mathrm{NaCl}$ into concentrations of $10^{-1}$.

Electron microscopy and SDS-PAGE. Viral-infected cell cultures were fixed at $4^{\circ} \mathrm{C}$ with $2.5 \%$ glutaraldehyde in phosphate buffer $(0.1 \mathrm{M}), \mathrm{pH} 7.2$, and post-fixed in $1.0 \%$ osmium tetroxide (OsO4). After another rinsing with phosphate buffer, the specimens were dehydrated in graded ethanol and embedded in Epon 812 (Epikote resin). Ultrathin sections were sliced with glass knives on a UCT ultramicrotome (Leica Ltd, Germany), stained with uranyl acetate and lead citrate, and examined under a Tecnai 12 electron microscope (FEI, Acht, The Netherlands). 
Viral-infected cell cultures were frozen at $-20^{\circ} \mathrm{C}$, and thawed at room temperature, followed by centrifugation for $30 \mathrm{~min}$ at $1,500 \times \mathrm{g}$ to remove the cell fragments. The supernatant was collected as a viral solution and centrifuged at $10,500 \times \mathrm{g}$ for $2 \mathrm{~h}$ at $4^{\circ} \mathrm{C}$.

TRIZOL reagent (Invitrogen, USA) was used to extract dsRNA from infected CIK cells, according to the manufacturer's protocol. RNA quality was assessed by electrophoresis on $1 \%$ agarose gel, and the total RNA concentration was determined by measuring the absorbance at $260 \mathrm{~nm}$ on the spectrophotometer (Eppendorf, Germany). Extracted RNA was kept at $-70^{\circ} \mathrm{C}$. Viral dsRNA was analyzed by SDS-PAGE on vertical slab gels ( $8 \%$ polyacrylamide gel) in Laemmli's buffer, and then visualized by silver staining.

Reverse transcription-polymerase chain reaction (RT-PCR). A pair of specific primers (GD-S9F: 5'-CTTTGAGTCGACGCACGTAT-3'， GD-S9R: 5'-CCGTCGGGTGGATTAGGT-3') were designed to amplify complete CDS of S9 gene (coding of VP6 protein) of eight typical GCRV isolates. Virus CDNA was constructed by reverse transcription with PrimeScriptTM RT reagent Kit (Takara, China) following the supplier's instructions. The resulting CDNA was amplified by PCR with the specific primers, and the PCR reaction was performed for 35 cycles of $94^{\circ} \mathrm{C}$ for $40 \mathrm{~s}, 59^{\circ} \mathrm{C}$ for $40 \mathrm{~s}, 72^{\circ} \mathrm{C}$ for $1 \mathrm{~min} 20 \mathrm{~s}$, and followed by a final extension at $72^{\circ} \mathrm{C}$ for $10 \mathrm{~min}$. The amplified PCR products were subjected to gel electrophoresis and observed under UV light.

Cloning and sequencing. Each PCR product from different isolates were ligated into a PMD-19T vector (Takara,China), and transformed into E.coli DH5a competent cells. Positive clones were screened via blue-white selection and detected by PCR with M13 \pm primers. After all the bacteria liquid was sequenced by Sangon Bothech (Shanghai) Co., Ltd. Sequence accession numbers were deposited in GenBank (KC130075KC130082).

Phylogenetic analysis of the CDS of S9 gene. The complete sequences of the S9 gene were translated and compared with amino acid sequences of the viral coat protein from the other variant members of Reoviridae. Multiple sequence alignments were carried out using Lasergene 7.1 software (DNASTAR Inc., Madison, WI). The unrooted phylogenetic tree was generated by neighbor-joining method using MEGA 4.0.

Amino acid analysis of VP6. To explore the genetic variation of the GCRV samples, amino acid sequences of VP6 were analyzed from eight isolates, compared with some similar isolates in previous studies. In particular, the primary neutralizing epitope (PNE) of VP6 were predicted and analyzed using Lasergene 7.1 and Goldkey software.

\section{Results}

Epidemiological and geographical features of GCRV in China. In the Yangtze River and Pearl River basin, epidemic outbreaks of GCRV occur June-September every year, causing serious harm mainly to fingerling and yearling grass carp. In this research, 82 positive samples of GCRV were detected by primer $1 ; 7$ positive samples were detected by primer 2 ; and 1 sample was detected by both primers. The naturally diseased fish exhibited typical hemorrhagic symptoms with severe mortality. The pathological changes associated with cases of death included foci with pathological changes and ischemia in the liver together with small intestine hemorrhagic spots, and edema, blood spots in kidney, and hemorrhagic spots in muscle.

Virus culture, electron microscopy and SDS-PAGE analysis. The virus from eight typical samples could be propagated in the grass carp cell line, CIK. None of them caused 
obvious cytopathic effects, however after intraperitoneal injection with frozen and thawed cell suspension, they did. Healthy grass carp suffered $80-100 \%$ mortality with hemorrhagic symptoms, similar to those in the naturally diseased fish.

Under electron microscopy, spherical virus was arranged in a honeycomb shape. They were about $75 \mathrm{~nm}$ in diameter with a similar size $(70-90 \mathrm{~nm})$ as AQRVs. The evident boundary displayed a prominent white ring in the negatively stained electron micrograph between the outer capsids and inner cores, (Fig. 1). Significant variations were observed in the genomes of GCRV-YX, GCRV-ZS, and GCRV873, and were analyzed by SDS-PAGE though both genome segments were separated into 11 distinct bands (Fig. 2).

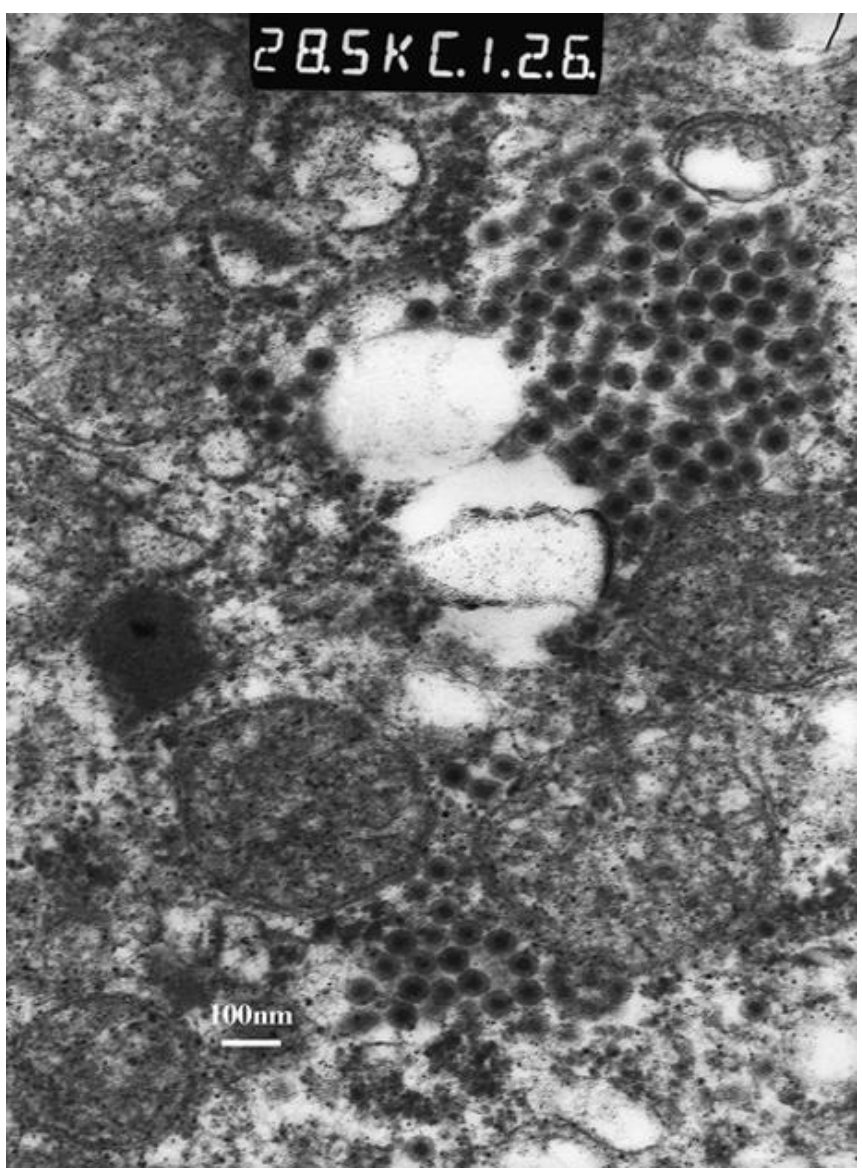

Fig. 1. Viral particles in CIK cell. The scale bar represents $100 \mathrm{~nm}$ 


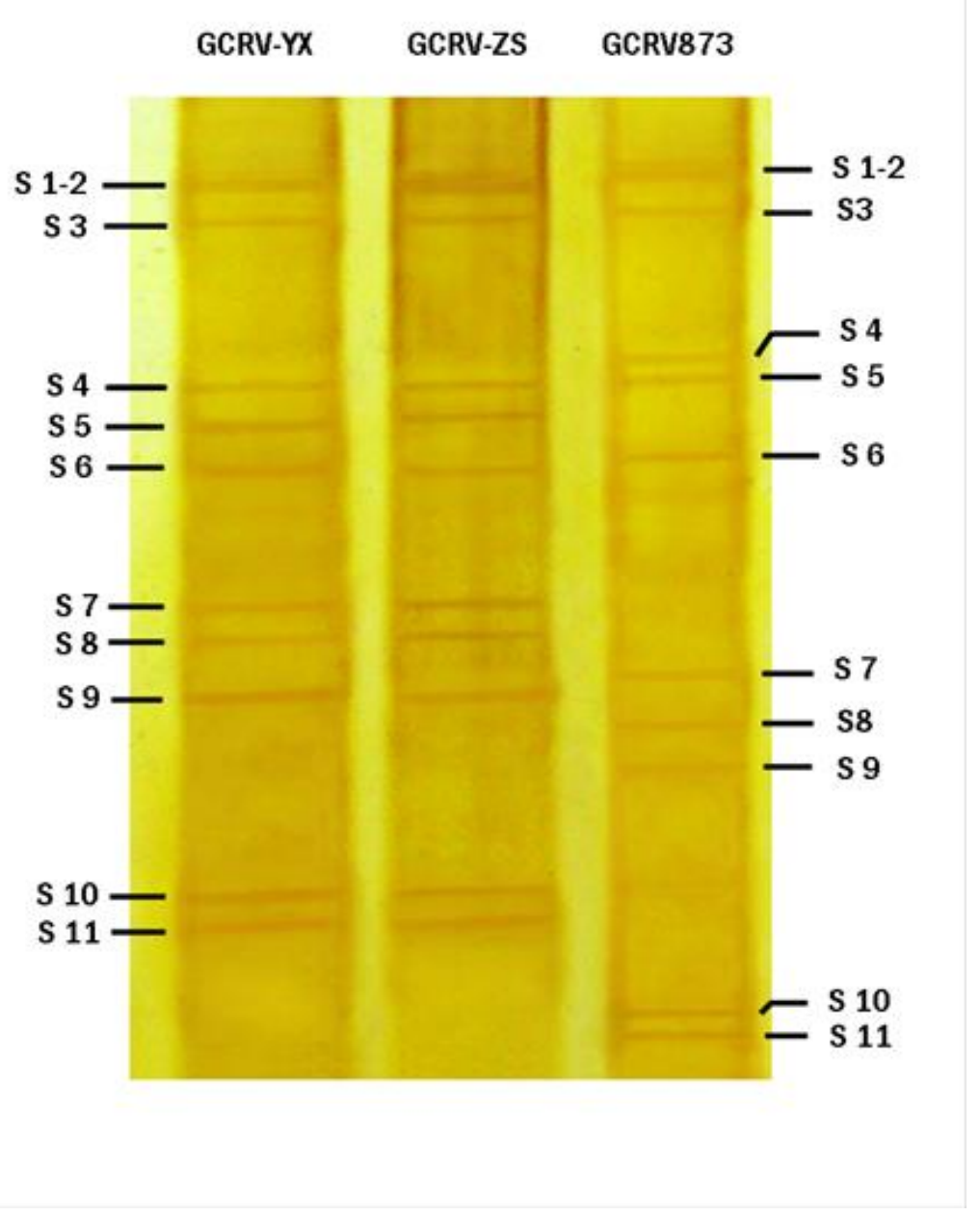

Fig. 2. Genome of three virus strains by SDS-PAGE

Sequence and phylogenetic analyses of S9 gene. The complete CDS sequences with $1257 \mathrm{bp}$ of S9 gene from the eight GCRV isolates that displayed high homologies, shared $97.1-100 \%$ nucleotide and $97.6-100 \%$ amino acid sequence identities. The S9 gene sequences of the eight acute GCRV isolates shared 97.0-99.1\% nucleotide and 97.198.5\% amino acid sequence identities with GCRV GD-108 and GCRV HZ08. The eight sequences only shared $39.6-40.1 \%$ nucleotide and $21.6-22.5 \%$ amino acid sequence identities with GCRV873, 34.6-35.2\% nucleotide and 18.2-18.5\% amino acid sequence identities with GCRV 104 (Table 1). 
Table 1. Nucleotide and deduced amino acid identities of GCRV S9 gene sequences.

\begin{tabular}{|c|c|c|c|c|c|c|c|c|c|c|c|c|c|c|c|c|}
\hline \multicolumn{17}{|c|}{ Nucleotide identity } \\
\hline & 1 & 2 & 3 & 4 & 5 & 6 & 7 & 8 & 9 & 10 & 11 & 12 & 13 & 14 & & \\
\hline 1 & & 99.1 & 99.0 & 99.0 & 97.8 & 99.0 & 99.2 & 97.5 & 98.8 & 97.0 & 35.2 & 39.0 & 39.6 & 40.1 & 1 & GCRV-HN \\
\hline 2 & 99.0 & & 99.7 & 99.7 & 98.0 & 99.7 & 99.9 & 97.2 & 99.0 & 97.2 & 35.2 & 39.1 & 39.7 & 40.2 & 2 & GCRV-HS \\
\hline 3 & 100.0 & 99.0 & & 99.9 & 97.9 & 99.9 & 99.7 & 97.1 & 98.8 & 97.0 & 35.2 & 39.0 & 39.6 & 40.1 & 3 & GCRV-JS \\
\hline 4 & 99.7 & 99.2 & 99.7 & & 98.0 & 100.0 & 99.8 & 97.2 & 98.9 & 97.1 & 35.2 & 39.0 & 39.6 & 40.1 & 4 & GCRV-NC \\
\hline 5 & 97.8 & 98.3 & 97.8 & 98.0 & & 98.0 & 98.1 & 99.2 & 97.6 & 98.3 & 34.9 & 39.0 & 40.1 & 40.5 & 5 & GCRV-QC \\
\hline 6 & 99.7 & 99.2 & 99.7 & 100.0 & 98.0 & & 99.8 & 97.2 & 98.9 & 97.1 & 35.2 & 39.0 & 39.6 & 40.1 & 6 & GCRV-QY \\
\hline 7 & 99.2 & 99.7 & 99.2 & 99.5 & 98.5 & 99.5 & & 97.3 & 99.1 & 97.2 & 35.2 & 39.0 & 39.6 & 40.1 & 7 & GCRV-YX \\
\hline 8 & 97.6 & 98.0 & 97.6 & 97.8 & 99.7 & 97.8 & 98.3 & & 97.2 & 99.1 & 34.6 & 38.8 & 40.1 & 40.4 & 8 & GCRV-ZS \\
\hline 9 & 97.8 & 98.3 & 97.8 & 98.0 & 98.0 & 98.0 & 98.5 & 97.8 & & 97.2 & 34.9 & 38.9 & 39.6 & 40.1 & 9 & GD108 \\
\hline 10 & 97.1 & 97.6 & 97.1 & 97.3 & 98.3 & 97.3 & 97.8 & 98.5 & 97.3 & & 34.7 & 38.8 & 40.0 & 40.4 & 10 & HZO8 \\
\hline 11 & 18.2 & 18.2 & 18.2 & 18.2 & 18.3 & 18.2 & 18.3 & 18.5 & 18.0 & 18.2 & & 35.1 & 37.0 & 37.0 & 11 & GCRV104 \\
\hline 12 & 20.1 & 20.3 & 20.1 & 20.1 & 20.6 & 20.1 & 20.5 & 20.6 & 20.6 & 20.6 & 15.4 & & 62.5 & 62.5 & 12 & AGCRV \\
\hline 13 & 22.3 & 22.5 & 22.3 & 22.3 & 22.0 & 22.3 & 21.6 & 22.0 & 22.0 & 22.3 & 15.9 & 51.9 & & 94.6 & 13 & GCRV873 \\
\hline 14 & 22.3 & 22.5 & 22.3 & 22.3 & 22.0 & 22.3 & 21.8 & 22.0 & 22.1 & 22.3 & 15.9 & 51.9 & 98.7 & & 14 & GSRV \\
\hline \multicolumn{17}{|c|}{ Amino acids identity } \\
\hline
\end{tabular}

To further understand the genetic evolution relationship of acute GCRV, phylogenetic analysis for the sequences of the VP6 gene included eight acute GCRV isolates similar to other reovirus reported worldwide (Fig.3). There are three distinct genotypes from the NJ-tree: subgroup1 (SG1) represented by GCRV GD108 belong to genotype II, SG2 represented by GCRV 873 belong to genotype I and SG3 represented by GCRV 104 belong to genotype III. 


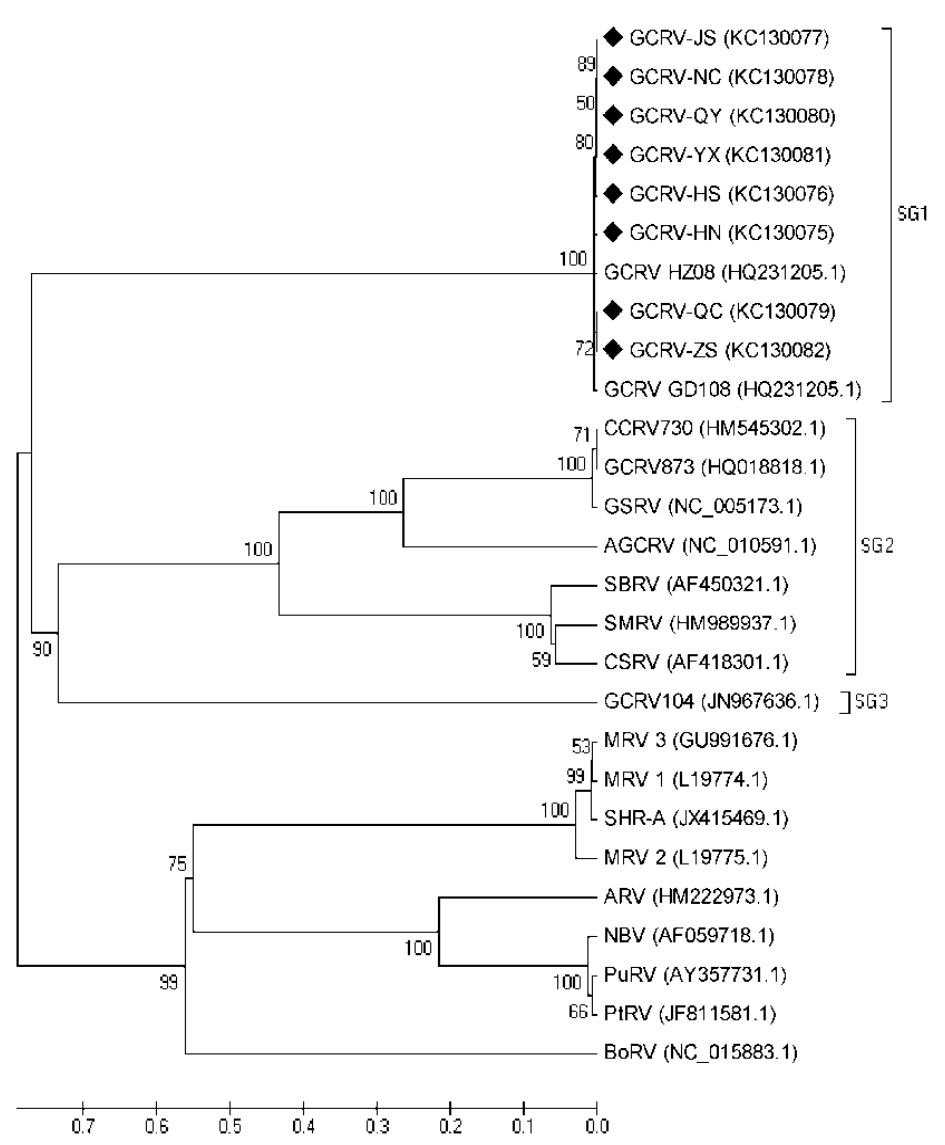

Fig. 3. Phylogenetic tree was constructed using Neighbor-Joining method based on VP6 sequences of Aquareovirus and Orthoreovirus. The reliability of the tree was assessed by bootstrapping it with 1000 replications. The black rhombus stands for our isolates in this study.

Amino acid analysis of VP6 protein. The protein VP6 included 418 amino acids, which contain a rich potential epitope region. The hydrophilicity, antigenic index, surface probability, and flexibility, were analyzed to find some probable epitope regions (Table 2). Variations of all epitope regions were highly conserved among isolates of SG1, which had no amino acid mutation. But compared with genotype I and genotype II, they showed marked differences, and no similar peptides in these probable epitope regions.

Table 2. The epitopes of VP6 in GCRV

\begin{tabular}{ll|ll}
\hline \multicolumn{2}{c|}{ genotype II } & \multicolumn{2}{c}{ genotype / } \\
\hline Epitope sequence & Amino acid position & Epitope sequence & Amino acid position \\
\hline QARPDGNV & $164-171$ & RPWTSRFS & $35-42$ \\
FPKKRKFED & $237-245$ & GRLNRSRTC & $221-229$ \\
ATRDEDD & $372-378$ & QWRQDGLI & $340-347$ \\
DDRCCCLKP & $386-394$ & QEAQLNNDYTA & $351-361$ \\
\hline
\end{tabular}

\section{Discussion}

Although extensive research about GCRV has been available, hemorrhage disease of grass carp still frequently occurs due to lack of accurate and timely epidemiological study. In our study, epidemiological surveys were executed in the Yangtze River and Pearl River Basin, where there were outbreaks of hemorrhage disease of grass carp. 
Yang et al.

Among the positive samples, more than $90 \%$ belonged to genotype II. However, prior research on GCRV was on genotype I while the current major epidemic strain has rarely been reported (Chao et al. 2010; Qing Wang et al.2012; Zeng et al. 2011). It is necessary to investigate genotype II for better understanding of the epidemiology of GCRV.

Protein VP6 of GCRV was recognized as the counterpart of protein Sigma2 of MRV occupying similar positions as the nodules on the surface of the inner capsid (Zhou et al. 2013). A blast analysis showed that VP6 of GCRV GD-108 possesses conserved domains belonging to the reoviral Sigma1 and Sigma2 superfamily. The reoviral Sigma1 and Sigma2 proteins consist of two concentric icosahedral layers in the inner core and the outer capsid layer (Dermody et al. 1991; Sellers et al. 2004). These studies revealed that VP6 protein has a close correlation with the viral capsid. In our experiments isolates of genotype II displayed high similarities with each other, with a high sequence homology. However, they also exhibited differences compared with other species of AQRV, including the genotype I and genotype III. Although both genotype I and genotype II of GCRV cause similar hemorrhagic symptoms, they also differ for culturing in Cytokine-induced killer cells (CIK). For genotype I, the cytopathic effects typical of AQRV infections were observed in cells cultured for $48 \mathrm{~h}$, but genotype II was not, even after three generations. Furthermore, although all strains of GCRV possessed 11 dsRNA fragments, the VP6 gene did not exhibit high homology between these three strains (only 21.6-22.5\%). Similar results also occurred in VP5, the homology between GCRV GD-108 and other AQRVs was only 24-25\% (Ye et al. 2012). Finally, based on sequence analysis, we found that these two genotypes of GCRV did not have immunological cross-reactions.

The exact epidemiologic relationship of GCRV is difficult to establish as different isolates belong to two distinct sub-populations. Research suggests that GCRV-GD108 might fill some of the evolutionary gaps between Aquareovirus and Orthoreovirus as it shares a closer phylogenetic relationship with ORV than other AQRVs. Undoubtedly the incidence of genotype I has decreased yearly and genotype II has increased (Qing Wang et al.2012; Yang et al. 2010; Zhang et al. 2010). In summary, the existence of two genotypes of GCRV detected, and genotype II was the prevalent strain in South China. Considering the complex variations of GCRV, there may be more genotypes which have not been reported or detected, so the scientific classification of GCRV is a prerequisite. Extensive genetic diversity must be associated with these outbreaks. The abundant genetic diversity should be taken into consideration to control and prevent this disease.

\section{Acknowledgments}

This work was jointly supported by the National Spark Program Project of China (2015GA780004) and Guangdong Natural Science Foundation (2013B020503070). We would like to thank Yu-dong Du (Project manager of Hold-one aquatic breeding co., LTD) for providing samples.

\section{References}

Bo X., Chi X., Zhang L., Qu H., Liu Y., Wang X. and J. Zhou, 2014. Enhanced expression of GCRV VP6 in CIK cells by relative sequence optimization. Appl Biochem Biotechnol. 173 (8): 2129-2139.

Chao Z., Wang Q., Shi C., Zeng W., Liu Y. and S. Wu, 2010. Molecular analysis of grass carp reovirus HZ08 genome segments 1-3 and 5-6. Virus Genes. 41 (1): 102-104.

Dermody T.S., Schiff L.A., Nibert M.L., Coombs K.M. and B.N. Field, 1991. The S2 gene nucleotide sequences of prototype strains of the three reovirus serotypes: characterization of reovirus core protein sigma 2. J Virology. 65 (11): 5721-5731. 
Huan., Xiaoming. and Q. Fang, 2014. The VP2 protein of grass carp reovirus (GCRV) expressed in a baculovirus exhibits RNA polymerase activity. Virologica Sinica. 29 (2): 86-93.

Iwanowicz L.R., Iwanowicz D.D., Adams C.R., Lewis T.D., Brandt T.M., Cornman R.S. and L. Sanders, 2016. Complete Genome Sequence of a Novel Aquareovirus That Infects the Endangered Fountain Darter,Etheostoma fonticola. Genome Announcements. $4(6)$.

Jia R., Cao L.P., Du J.L., Liu Y.J., Wang J.H., Jeney G. and G.J. Yin, 2014. Grass carp reovirus induces apoptosis and oxidative stress in grass carp (Ctenopharyngodon idellus) kidney cell line. Virus Res. 185 (25): 77-81.

Liang H.R., Fu X.Z., Li N.Q., Liu L.H., Lin Q., Li Y.G., Peng Y.A., Huang Z.B. and S.Q. Wu, 2016. The distribution of different virulence grass carp reovirus strains in some neglected tissues. Polish J Vet Sci. 19 (4): 763.

Qing Wang., Zeng W., Liu C., Zhang C., Wang Y., Shi C. and S. Wu, 2012. Complete Genome Sequence of a Reovirus Isolated from Grass Carp, Indicating Different Genotypes of GCRV in China. J Virol. 86 (22): 12466.

Sellers H.S., Linnemann E.G., Pereira L. and D.R. Kapczynski, 2004. Phylogenetic analysis of the sigma 2 protein gene of turkey reoviruses. Avian Diseases. 48 (3): 651-7.

Tian Y., Ye X., Zhang L., Deng G. and Y. Bai, 2013. Development of a novel candidate subunit vaccine against Grass carp reovirus Guangdong strain (GCRV-GD108). Fish \& Shellfish Immunol. 35 (2): 351-356.

Wang H., Ye X., Tian Y., Zhang L. and G. Deng, 2013. Analysis of function and immunogenicity of GCRV-GD108 VP5. J Fish China. 37 (1): 109.

Yang X.U., Hao G.J., Shen J.Y., Zheng S.J., Pan X.Y., Yao J.Y. and W.L. Yin, 2010. Isolation and identification of two grass carp reovrius strains in Jiangxi province. Freshwater Fisheries. 40 (3): 44-49.

Ye X., Tian Y.Y., Deng G.C., Chi Y.Y. and X.Y. Jiang, 2012. Complete genomic sequence of a reovirus isolated from grass carp in China. Virus Res. 163 (1): 275-283.

Zeng W.W., Wang Q., Liu Y.K., Zhang L.S., Liu B.Q., Shi C.B. and S.Q. Wu, 2011. Isolation and identification of new GCRV strain and primary study on its immunogenicity. Acta Hydrobiologica Sinica. 35 (5): 790-795.

Zeng W., Yao W., Wang Y., Li Y., Bermann S.M., Ren Y., Shi C., Song X., Huang Q. and S. Zheng, 2017. Molecular detection of genotype II grass carp reovirus based on nucleic acid sequence-based amplification combined with enzyme-linked immunosorbent assay (NASBA-ELISA). J Virological Methods. 243: 92-97.

Zhang L., Luo Q., Qin F. and Y. Wang, 2010. An improved RT-PCR assay for rapid and sensitive detection of grass carp reovirus. J Virological Methods. 169 (1): 28-33.

Zhou Y., Fan Y.D., Zeng L.B. and J. Ma, 2013. Prokaryotic expression and immunoassay of grass carp reovirus capsid VP6 protein. Acta virologica. 57 (4): 456. 